\title{
Mortalidade infantil na percepção de gestores e profissionais de saúde: determinantes do seu declínio e desafios atuais em município do sul do Brasil'
}

\author{
Infant mortality in the perception of health managers and \\ professionals: determinants of its decline and current \\ challenges in a southern Brazilian town
}

\author{
Lígia Goes Pedrozo Pizzo \\ Mestre em Saúde Coletiva. Enfermeira do município de Cambé. \\ Endereço: Rua Benjamin Franklin, 360, casa 16, CEP 86063-240, \\ Londrina, PR, Brasil. \\ E-mail: liginhaenfळyahoo.com.br \\ Selma Maffei de Andrade \\ Doutora em Saúde Pública. Professora Associada do Departamento \\ de Saúde Coletiva da Universidade Estadual de Londrina. \\ Endereço: Av. Robert Koch, 60, CEP 86038-350, Londrina, PR, Brasil. \\ E-mail: selmaffeiळgmail.com

\section{Ana Maria Rigo Silva} \\ Doutora em Saúde Pública. Professora Adjunta do Departamento \\ de Saúde Coletiva da Universidade Estadual de Londrina. \\ Endereço: Av. Robert Koch, 60, CEP 86038-350, Londrina, PR, Brasil. \\ E-mail: arigosilva®gmail.com

\section{Regina Melchior} \\ Doutora em Saúde Pública. Professora Associada do Departamento \\ de Saúde Coletiva da Universidade Estadual de Londrina. \\ Endereço: Av. Robert Koch, 60, CEP 86038-350, Londrina, PR, Brasil. \\ E-mail: reginamelchior@gmail.com

\section{Alberto Durán González} \\ Doutor em Saúde Coletiva. Professor Adjunto do Departamento de \\ Saúde Coletiva da Universidade Estadual de Londrina. \\ Endereço: Av. Robert Koch, 60, CEP 86038-350, Londrina, PR, Brasil. \\ E-mail: betoduran8oळgmail.com

\footnotetext{
I Esta pesquisa contou com auxílio financeiro do Decit/SCTIE/ Ministério da Saúde, por intermédio do Conselho Nacional de Desenvolvimento Científico e Tecnológico (CNPq) e da Fundação de Nível Superior (CAPES) e bolsa produtividade em pesquisa do Conselho Nacional de Desenvolvimento Científico e Tecnológico
} \\ Araucária (Chamada 08/2009 da Fundação Araucária - PPSUS), \\ bolsa de mestrado da Coordenação de Aperfeiçoamento de Pessoal \\ (CNPq).
}

\section{Resumo}

Este estudo objetivou analisar a percepção de gestores e profissionais da saúde sobre os determinantes para o declínio da mortalidade infantil nas últimas décadas, bem como os desafios para a continuidade da redução dos valores desse indicador em Londrina (PR). Foi realizado uma pesquisa qualitativa com análise de entrevistas de 38 gestores, profissionais de saúde e pesquisadores, selecionados pela técnica da bola de neve. Na visão dos entrevistados, os determinantes para a redução da mortalidade infantil foram melhorias das condições de vida e medidas implantadas por políticas públicas e ações setoriais e extrassetoriais. Os desafios atuais dizem respeito, principalmente, à qualificação da assistência pré-natal, à redução da gravidez na adolescência, à melhoria da atenção hospitalar ao recém-nascido prematuro, à prevenção da prematuridade e ao financiamento do Sistema Único de Saúde (SUS). 0 aumento da proporção de mortes neonatais torna mais complexo e difícil reduzir a mortalidade infantil. Compreender as dificuldades e identificar os desafios, sob a percepção de gestores e profissionais da saúde, pode contribuir para o planejamento das ações necessárias para a melhoria da saúde das crianças menores de 1 ano e para a redução da mortalidade infantil.

Palavras-chave: Mortalidade Infantil; Saúde Materno-infantil; Pesquisa Qualitativa. 


\section{Abstract}

This study aimed to analyze the perception of health managers and professionals of determinants for the decline in infant mortality over the last decades, as well as the challenges to keep on reducing the values of this indicator in Londrina, Paraná, Brazil. A qualitative research was conducted by analyzing the interviews of 38 managers, health professionals, and researchers, selected using the so-called snowball technique. According to respondents' view, the determinants for reducing infant mortality were improvements in life conditions and measures implemented through public policies and sector and extra-sector actions. The current challenges are mainly related to providing a better prenatal care, reducing adolescent pregnancy, improving the hospital care provided to a premature newborn infant, prematurity prevention, and funding by the Brazilian National Health System. The increased proportion of neonatal deaths makes reducing infant mortality more complex and difficult. Understanding difficulties and identifying challenges, in the perception of health managers and professionals, may contribute to planning the actions needed to improve the health of children under 1 year of age and reduce infant mortality. Keywords: Infant Mortality; Maternal and Child Health; Qualitative Research.

\section{Introdução}

A taxa de mortalidade infantil (TMI) é indicadora das condições de vida e de saúde de uma população porque expressa não somente causas biológicas, mas, principalmente, determinações de ordem socioeconômica e ambiental (Vermelho e col., 2009). No Brasil, essa taxa reduziu-se de 47 para 20 por mil nascidos vivos de 1990 a 2007, com diminuição das disparidades regionais, porém com desaceleração na velocidade de seu declínio após os anos 2000 (Barros e col., 201ob). Em Londrina, Paraná, também houve queda constante da TMI até 1997. Em anos mais recentes, observa-se estagnação nos valores desse indicador, mantendo-se, desde 2005, em torno de 9 a 12 óbitos por mil nascidos vivos (Londrina, 2011).

Estudos sobre a mortalidade na infância são imprescindíveis para análise do nível de saúde e para avaliação do impacto de intervenções, havendo no Brasil várias investigações epidemiológicas sobre o tema (Duarte, 2007). Porém, pesquisas qualitativas também são fundamentais para melhor compreensão do objeto estudado, pois trazem à luz percepções, representações, significados e perspectivas dos sujeitos que participam da pesquisa (Turato, 2005), podendo contribuir para a adoção de políticas e estratégias apropriadas. Todavia, poucas pesquisas qualitativas brasileiras com foco na mortalidade infantil ou em perdas fetais foram identificadas na literatura (Goulart e col., 2005; Machado e col., 2011; Nations, 2008; Santos e col., 2004; Sousa e Nations, 2011), a maior parte tendo como foco a perspectiva das mães que vivenciaram a experiência de perda de um filho.

Nesse sentido, o presente estudo teve por objetivo identificar a percepção de profissionais e gestores que atuam na atenção à saúde de mulheres e crianças sobre os determinantes para o declínio da TMI nas últimas décadas, bem como os desafios para a continuidade de redução dessa taxa no município de Londrina, conforme referido, com taxas estabilizadas de mortalidade infantil nos últimos anos.

\section{Trajetória metodológica}

O estudo, com abordagem qualitativa, foi desenvolvido em Londrina, Paraná, município que contava com 506.645 habitantes em 2010 (Datasus, 2011). 
Sua rede básica de saúde está organizada para atender gestantes e crianças em 52 unidades básicas de saúde (UBS). A assistência ao parto era prestada, à época do estudo, por cinco hospitais, dos quais dois privados e três vinculados ao Sistema Único de Saúde (SUS) - um filantrópico e dois públicos. Três hospitais proporcionam atenção intensiva ao recém-nascido (RN), todos vinculados ao SUS (dois filantrópicos e um público). 0 município foi um dos pioneiros na expansão e descentralização de serviços universais de atenção primária à saúde no final da década de 1970 (Gil e col., 2001).

Os participantes do estudo foram profissionais de saúde que atuavam ou atuaram na assistência materno-infantil como gestores do sistema municipal de saúde (secretários municipais ou dirigentes) e como docentes/pesquisadores da temática. Inicialmente, com base na experiência de duas pesquisadoras, que são profissionais que já atuaram nos serviços de atenção básica e em cargos de direção do serviço de saúde municipal, foi elaborada uma lista contendo 21 nomes de pessoas consideradas "informantes-chave".

As entrevistas das pessoas listadas foram conduzidas com apoio de um roteiro semiestruturado, previamente testado, que continha questionamentos a respeito dos fatores que contribuíram para a redução da mortalidade infantil nas últimas décadas. Foram investigadas, também, ações que precisariam ser aperfeiçoadas ou implantadas para acentuar a redução dos óbitos infantis ou que eram vistas como desafios a essa redução.

Por meio da técnica bola de neve (Dunn e Ferri, 1999), os 21 informantes inicialmente listados podiam indicar outros sujeitos que considerassem ter algo a contribuir com a pesquisa, e os indicados também poderiam sugerir outras pessoas. Nesse processo, foram sugeridos mais 30 profissionais, totalizando 51, porém foram entrevistados 39, por saturação dos dados (Fontanella e col., 2008). A coleta de dados ocorreu de abril a julho de 2009, e os depoimentos foram gravados com aparelho de áudio e transcritos.

Uma pessoa concedeu entrevista, mas não aceitou a gravação, sendo excluída da análise. Assim, foram consideradas 38 entrevistas. Foi adotada a técnica de análise de conteúdo, com leituras flu- tuantes e exaustivas das entrevistas transcritas, destaque das unidades de registro e definição das categorias de análise (Bardin, 2004). A leitura das transcrições e a definição das categorias de análise foram feitas por duas pesquisadoras e discutidas entre todos os autores, até definição final.

Os participantes foram identificados segundo a função profissional que motivou a sua inclusão no estudo, embora a maioria tivesse exercido mais de uma função. Todos os entrevistados, exceto dois, haviam exercido ou exerciam assistência direta à saúde de crianças ou gestantes/parturientes. Os que foram selecionados porque tinham ou tiveram cargo técnico de nível central foram identificados com a letra C; os que atuavam exclusivamente na área assistencial, com a letra A; os gestores, com G; e os pesquisadores/docentes do ensino superior, com $\mathrm{P}$.

O projeto de pesquisa foi aprovado pelo Comitê de Ética da Universidade Estadual de Londrina. Todos os entrevistados assinaram termo de consentimento livre e esclarecido, após explicações sobre a pesquisa.

Os discursos sobre a redução da mortalidade infantil foram agrupados em três categorias: a) condições de vida e políticas públicas gerais; b) políticas/ ações setoriais; e c) ações extrassetoriais. A análise dos discursos sobre os desafios resultou em quatro categorias: a) qualificação da atenção pré-natal; b) gravidez na adolescência; c) atenção ao recém-nascido prematuro e prevenção da prematuridade; e d) outros desafios setoriais.

\section{Resultados e discussão}

Dos 38 participantes do estudo, 12 foram classificados como gestores, 14 como técnicos de nível central, 9 como pesquisadores e 3 como com atuação exclusiva na assistência à saúde da mulher e/ou da criança. Vinte e sete eram mulheres, das quais 14 enfermeiras, 12 médicas e 1 dentista. Os 11 homens eram todos médicos.

\section{Determinantes da redução da mortalidade infantil}

Na percepção dos entrevistados, a melhoria nas condições de vida e as políticas públicas gerais fo- 
ram determinantes para a redução da mortalidade infantil, a partir dos anos 1970 até recentemente.

De 1960 a 72 houve uma melhora do nível de saúde [...] O primeiro fator foi a expansão da rede de água [...] De zooo até hoje, há uma melhoria muito importante da condição econômica [...] Não é uma política de saúde, mas tem peso na saúde $\left(\mathrm{P}_{3}\right)$.

Políticas públicas gerais e compensatórias também foram salientadas, como a ampliação da licença-maternidade de 90 para 120 dias, o Bolsa Família (G5), os programas nacionais e estaduais de fornecimento de leite, nos anos 1980 e a ampliação dessas políticas nos anos 1990 e 2000.

As políticas sociais também contribuíram. Se não contribuíram para a diminuição dos coeficientes, pelo menos para a estabilização deles (G8).

Em sintonia com a percepção dos entrevistados, o impacto da melhoria das condições de vida na redução da mortalidade infantil no Brasil, nas últimas décadas, tem sido relatado em vários estudos (Barros e col., 201ob; Costa e col., 2003; Hernandez e col., 2011; Holcman e col., 2004; Machado e col., 2011). Cabe destacar que essa redução se deu à custa, principalmente, do componente pós-neonatal da mortalidade infantil, período de vida em que as condições socioeconômicas, de nutrição, meio ambiente e atenção primária à saúde exercem papel relevante na saúde infantil (Tomé e Latorre, 2001; Vermelho e col., 2009). As condições concretas de vida e as desigualdades sociais estão bem documentadas na literatura como determinantes para o maior risco de morte infantil (Cruz e col., 2011; Zanini e col., 2009), porém alguns estudos revelam que prevalece, ainda, entre profissionais e gestores da saúde uma visão que reduz o início da cadeia causal do evento à família e que culpabiliza a mãe que perdeu seu filho (Goulart e col., 2005; Sousa e Nations, 2011). Essa percepção de culpabilização da mãe ou das famílias, porém, não foi evidenciada no presente estudo.

Na categoria políticas/ações setoriais, a organização da rede municipal de saúde, em especial da atenção básica de acesso universal, nos anos 1970 e 1980, anteriormente à constituição do SUS, foi enfatizada pelos profissionais do estudo.

Uma ação importante foi a implantação da rede municipal de saúde, a descentralização dos ser- viços desde 1978 (quando foram implantados os primeiros postos de saúde), foi se viabilizando uma rede de atenção $(\mathrm{P} 1)$.

Foram as ações básicas de saúde (nessa época): terapia de reidratação oral, imunização, aleitamento materno (P8).

Os entrevistados destacaram a implantação do Sistema Único de Saúde, no início dos anos 1990, ao qual foi atribuída a importância pela universalização do acesso à saúde e ampliação da rede básica.

A Estratégia Saúde da Família (ESF) foi fundamental, segundo os entrevistados, para a reorganização da assistência à mulher e à criança e proporcionou a ampliação de recursos humanos, o maior contato das equipes com as famílias e a busca ativa na comunidade. Estudos mostram melhor desempenho da ESF na assistência materno-infantil em comparação com as unidades de saúde tradicionais, embora ainda aquém das suas possibilidades e com necessidade de aperfeiçoamento de ações (Caldeira e col., 2010; Mendoza-Sassi e col., 2011). Outros pesquisadores registram que o maior impacto da ESF se dá em localidades com níveis mais elevados de mortalidade infantil, especialmente da pós-neonatal (Aquino e col., 2009).

Algumas ações específicas de atenção básica e hospitalar foram também percebidas como importantes. Avanços na oferta de exames laboratoriais durante o pré-natal, como urocultura de controle após tratamento contra infecção urinária e a perspectiva de implantação da pesquisa do Streptococcus agalactiae em gestantes na atenção básica, foram salientados.

Foi feita uma regulamentação interna recomendando que se fizesse a urocultura de controle após o tratamento. Um programa que ainda não está sistematizado no município, mas a UEL (Universidade Estadual de Londrina) já implantou,é a pesquisa do Streptococcus agalactiae na gestação $\left(\mathrm{C}_{4}\right)$.

Nos Estados Unidos, o Centers for Disease Control and Prevention (CDC) recomenda, desde 1996, o rastreamento universal do Streptococcus agalactiae em gestantes com 35 a 37 semanas de gestação, com antibioticoprofilaxia algumas horas antes do parto. Essa infecção tem sido a principal causa de septicemia neonatal precoce entre recém-nascidos 
norte-americanos, ainda que com redução nos últimos anos (Verani e col., 2010). No Brasil, no entanto, ainda não há protocolo nacional com diretriz sobre esse aspecto.

Recebeu destaque, pelos entrevistados, a iniciativa do serviço municipal de saúde de Londrina de implantar, com recursos próprios, a vacinação contra o Haemophilus influenzae tipo B (HIB) em 1996, anteriormente à sua inclusão no calendário nacional, em 1999. O impacto dessa intervenção específica foi avaliado em estudo que mostrou diminuição de $88,3 \%$ na incidência de meningite por HIB em menores de 5 anos no município, redução não observada nas demais cidades do Estado que não dispunham desse tipo de vacina (Takemura e Andrade, 2001).

Outras ações setoriais foram identificadas como importantes, e os entrevistados mencionaram ações de ensino, assistência e proteção do aleitamento materno (C1o), além de ações conjuntas entre o Comitê Municipal de Prevenção da Mortalidade Materna e Infantil (CMPMMI) e a Diretoria de Ações de Saúde para a inserção de medicações na relação municipal de medicamentos para o combate a infecções do trato urinário.

A cefalexina foi introduzida na cesta básica de medicamentos. [...] A gente começou a observar que os médicos estavam tratando gestantes com antibiótico que não cobria infecção causada por agente do trato urinário (C2).

A gente pactuou a priorização do aleitamento materno, independente de quem atendesse a criança. (A2).

O papel desempenhado pelos comitês municipais de incentivo ao aleitamento materno (CALMA), criado em 1994, e de prevenção da mortalidade materna e infantil (CMPMMI), implantado em 1999, também foi considerado relevante para a queda da mortalidade infantil. Estudos destacam a influência das ações desencadeadas em prol do aleitamento materno na redução da morbimortalidade de crianças (Bahl e col., 2005; Victora e col., 1987). Há que se mencionar que profissionais do município estudado têm relevante atuação nessa área, com realização de diversas pesquisas e implementação de ações que deram origem, inclusive, à Rede Amamenta Brasil, criada por portaria do Ministério da Saúde em novembro de 2008 (Souza, 2010).

Na percepção dos entrevistados, os sistemas de informação em saúde foram fundamentais para o monitoramento da mortalidade infantil, sobretudo a partir da municipalização do processamento dos dados e das ações de vigilância epidemiológica, em meados da década de 1990. Destacaram que a melhoria da informação é pré-requisito importante pra você nortear suas ações (G1).

Outros aspectos foram salientados pelos entrevistados, como a formação de recursos humanos proporcionada pelas instituições de ensino superior e, mais recentemente, o processo de educação permanente dos profissionais de saúde. Conforme os relatos, o CMPMMI proporciona espaço de educação permanente, pois há discussão com a equipe de saúde local sobre o processo que culminou no óbito infantil, uma estratégia que pode ser implantada em outras localidades. É possível que esse espaço de diálogo entre profissionais das unidades de saúde e do comitê deva-se a características específicas locais, que refletem a organização desse serviço, o qual, mais recentemente, tem investigado a totalidade dos óbitos infantis (Santos e col., 2012). Revela-se, com isso, no que diz respeito à investigação de mortes infantis, adesão superior à observada em outros municípios brasileiros (Mathias e col., 20o8; Venâncio e Paiva, 2010) e, possivelmente, maior empenho no esclarecimento da cadeia de eventos que levou à morte da criança.

Em relação à atenção hospitalar, o referenciamento com garantia de assistência a partos de alto risco e os avanços tecnológicos incorporados à área de atenção neonatal, como, por exemplo, respiração assistida, uso de corticóide antenatal e surfactante, foram outros fatores percebidos como contribuintes para a redução da mortalidade infantil. Apesar do aumento de leitos de UTI neonatal no Brasil, nos últimos anos, há que se ressaltar que ainda existem problemas no acesso e distribuição desses leitos (Barbosa e Cunha, 2011), sendo esse aspecto considerado ainda um desafio para a redução da TMI, como será abordado adiante.

A única ação extrassetorial percebida como importante pelos entrevistados foi a atuação da Pastoral da Criança na formação de lideranças nas 
comunidades para o acompanhamento das crianças em situação de risco, especialmente em décadas anteriores. A importância da entidade também foi percebida por médicos do Programa Saúde da Família em Duque de Caxias (RJ) substituir por (Silva e col., 2009a), com destaque à aliança da pastoral com a unidade de saúde por meio de encaminhamentos, utilização do espaço da igreja, atuação conjunta no dia da pesagem e na recuperação nutricional das crianças, revelando parcerias que podem ser estabelecidas em cada comunidade.

\section{Desafios}

Na percepção dos entrevistados, um dos principais desafios para reduzir a mortalidade infantil está relacionado à qualificação da atenção pré-natal. A ausência de registros adequados em prontuários e problemas na comunicação entre profissional e gestante também foram mencionados como falta de qualidade.

Elas (gestantes), tendo seis consultas de pré-natal, não podiam estar com infecção urinária, não podiam estar com bebê com sífilis congênita! (G2).

Falta registro da altura uterina no pré-natal, faltam orientações, tanto por parte dos médicos quanto da enfermagem [...] Eu acho um absurdo uma gestante sair sem orientação... (C11).

Esses desafios também foram mencionados em outros estudos, que evidenciam que, apesar da boa cobertura da atenção pré-natal, ainda é preciso investir na melhoria da sua qualidade, especialmente no setor público (Domingues e col., 2012; Victora e col., 2010). Assim, revela-se a importância de investimentos para aprimorar a qualidade da atenção, motivar e capacitar profissionais de saúde para uma adequada adesão aos protocolos existentes e promover a articulação dos serviços de atenção básica e de assistência ao parto (Victora e col., 2011).

A gravidez na adolescência foi outro desafio percebido como a ser enfrentado, com destaque para a necessidade de avanço nas políticas intersetoriais (G8).

A vulnerabilidade de gestantes adolescentes e de seus filhos é destacada em alguns estudos quantitativos. Um deles (Oliveira e col., 2010) mostrou tendência de risco maior de morte infantil com a diminuição da idade materna, com efeito direto sobre os óbitos pós-neonatais e indireto sobre os neonatais. Outro trabalho revelou que do começo ao final dos anos 2000 houve redução das chances de morte de menores de 1 ano de idade filhos de mães adolescentes, porém a adolescência ainda se mantinha como fator de risco significativo para esse desfecho (Santos e col., 2012). No Brasil ocorreu grande redução da taxa de fecundidade nas últimas décadas, contudo essa taxa declinou mais lentamente entre adolescentes e aumentou discretamente entre meninas de 10 a 14 anos (Victora e col., 2011), o que torna o problema realmente merecedor de maior atenção.

Em Londrina houve redução da proporção de mães adolescentes nos últimos anos (Gabani e col., 2010). No entanto, a estreita relação da gravidez precoce com outros determinantes sociais e com indicadores de menor acesso e pior qualidade de atenção à saúde revelam que a vulnerabilidade dessas mães extrapola essa característica individual (Ferreira e col., 2012). Assim, é possível que a maior vulnerabilidade social de adolescentes grávidas tenha motivado a percepção dos entrevistados quanto a esse desafio.

A ampliação de leitos de unidades de terapia intensiva neonatal, sua melhor distribuição e a capacitação adequada de pessoal foram enfatizadas como desafios na categoria atenção ao recém nascido prematuro.

Ainda tem muito para avançar na questão da reorganização da distribuição de leitos de UTI neonatal [...] A gente tem uma qualidade questionável, porque está tendo abertura de novas UTI neonatais em função da falta de leitos, sem capacitação de pessoal adequado $\left(\mathrm{P}_{7}\right)$.

Ainda que se reconheça a importância das tecnologias disponíveis nessas unidades para aumentar a chance de sobrevida dessas crianças (Barros e col., 2010a), no Brasil o risco de mortalidade nesses serviços ainda é alto quando comparado a outros países (Gomes e col., 2005). Por isso, além de atenção adequada a esses recém-nascidos, há necessidade de se investir também em estudos que investiguem causas e em intervenções específicas que reduzam a prematuridade, fator mais diretamente relacionado à mortalidade neonatal (Gomes e col., 2005) . 
A taxa de prematuridade tem aumentado nos últimos anos, tanto no Brasil (Silveira e col., 2008) como em outros países (Martin, 2011). Em Londrina, observa-se uma das mais altas taxas de prematuridade do país (Silva e col., 20ogb), e esse fato pode ter contribuído para a percepção dos entrevistados quanto à necessidade de ampliação da qualidade da atenção hospitalar ao recém-nascido prematuro, com melhor distribuição de leitos de UTI e qualificação dos profissionais que atendem essas crianças.

Para a prevenção da prematuridade, os entrevistados do estudo entendem ser importante a regulação da reprodução assistida (G2), causa importante de gravidezes múltiplas e prematuridade (Graner e Barros, 2009).

Teria que ter algum acompanhamento desses mecanismos de fertilização, reprodução assistida. Precisava ter uma legislação que garantisse a qualidade, a sobrevida e o menor risco desses bebês, principalmente dessas gestações gemelares (G2).

Estudo caso-controle de base populacional realizado em Londrina evidenciou que mulheres que receberam "tratamento para engravidar" apresentaram risco bastante aumentado (cerca de oito vezes) para partos prematuros, mesmo controlando variáveis como local de residência (favela ou não), idade do responsável pelo sustento da família, índice de massa corporal da gestante e filho anterior pré-termo (Silva e col., 2009b). Em 2011, o Conselho Federal de Medicina revogou resolução do ano de 1992 que possibilitava a transferência de até quatro oócitos e embriões à receptora, e especificou que poderiam ser transferidos, no máximo, até dois para mulheres com até 35 anos, até três para mulheres entre 36 e 39 anos e até quatro para as com 40 anos ou mais (CFM, 2011). Essa nova resolução foi outra vez modificada em 2013, mantendo o mesmo número máximo de oócitos ou embriões passíveis de transferência de acordo com as idades, mas limitando-a em até 50 anos, além de estabelecer outras regras (CFM, 2013). Essas resoluções representam avanço em relação à situação anterior ao ano de 2011, porém ainda permanecem dificuldades relacionadas à supervisão dessas práticas.

Por fim, outros desafios setoriais, embora também dependentes do contexto político-econômico, foram postos: a carência de médicos pediatras (G2) disponíveis para atuar na atenção básica e a necessidade de prosseguimento no processo de educação permanente (G6).

A falta de pediatras no mercado possivelmente reflete as alterações no mundo do trabalho médico nos últimos anos, com seus avanços e incorporação de recursos tecnológicos e valorização de especialidades, além de fatores como a precarização de vínculos empregatícios, baixa remuneração e inadequada valorização profissional, contribuindo para a desmotivação de formandos em medicina pela especialidade (Bettiol, 2010; Junqueira e col., 2010). Há necessidade de se resgatar o papel desse profissional na saúde pública e ampliar o escopo de sua formação, objetivando não apenas a promoção da saúde e a prevenção/tratamento de doenças infantis, mas também a de doenças crônicas de adultos que se originam na infância (Bettiol, 2010).

Entre outras questões mais afetas ao setor saúde, destacou-se que o investimento em atenção primária à saúde precisa aumentar $\left(\mathrm{G}_{5}\right)$. 0 subfinanciamento do SUS é consenso entre pesquisadores da área, com destaque para a atenção básica, concordando com o depoimento apresentado. Noronha (2011) salienta que, em 2006, o gasto público per capita com saúde no Brasil limitava-se a US\$323, cerca de 10\% do observado nos Estados Unidos, e inferior ao de outros países da América do Sul, como Argentina (US\$ 549) e Colômbia (US\$ 396). Além disso, os principais gastos do SUS concentram-se em ações de média e alta complexidade, mais onerosas, especialmente em municípios maiores (Lima e Andrade, 2009).

\section{Considerações finais}

Em síntese, na visão dos profissionais desta pesquisa, a redução da mortalidade infantil nas últimas décadas decorreu de melhorias das condições de vida e medidas implantadas por políticas públicas e ações setoriais e extrassetoriais. Os desafios atuais percebidos referem-se à qualificação da assistência pré-natal, à redução da gravidez na adolescência, à melhoria da atenção ao recém-nascido prematuro, à prevenção da prematuridade, com destaque para o maior controle das técnicas de reprodução assistida e ao financiamento do SUS. Essa percepção é consoante com observações feitas por estudiosos 
do tema (Barros e col., 201ob; Victora e col., 2011). À medida que a mortalidade infantil se reduz, aumenta a proporção de mortes neonatais e se tornam mais complexas as ações necessárias ao seu enfrentamento, que passam a depender não só de melhorias nas condições de vida, mas também de maior investimento na infraestrutura dos serviços de saúde.

Salienta-se, ainda, que as altas taxas de cesarianas, tanto no Brasil (Barros e col., 2010b) como no município estudado, não foram reveladas nas falas dos profissionais do estudo como desafio à redução da mortalidade infantil, apesar de destacadas como um dos desafios em estudo que considerou o Brasil como um todo (Victora e col., 2011). Também não foi mencionada a necessidade de melhorar a qualidade da atenção durante o trabalho de parto, que tem se revelado precária em outros lugares do Brasil (Giglio e col., 2011; Oliveira e col., 2008) e cuja situação possivelmente não seja diferente em Londrina. Apesar disso, a percepção de trabalhadores e pesquisadores da área materno-infantil estudados foi coincidente em vários outros aspectos daquele estudo (Victora e col., 2011), revelando que esse tipo de pesquisa pode gerar conhecimento específico em cada localidade e contribuir para a adoção de ações que visem à melhoria da saúde e à redução da mortalidade infantil.

\section{Colaboração dos autores}

Pizzo participou da concepção do projeto, da coleta de dados, das análises dos resultados e da redação da primeira versão do artigo. Andrade e Rigo Silva participaram da concepção do projeto, das análises dos resultados e da redação e revisão crítica do artigo. Melchior e González participaram das análises dos resultados e da redação e revisão crítica do artigo. Todos os autores aprovaram a versão final do manuscrito.

\section{Referências}

AQUINO, R.; OLIVEIRA, N. F.; BARRETO, M. L. Impact of the family health program on infant mortality in Brazilian municipalities. American Journal of Public Health, Washington, DC, v. 99, n. 1, p. 87-93, 2009.
BAHL, R. et al. Infant feeding patterns and risks of death and hospitalization in the first half of infancy: multicentre cohort study. Bulletin of the World Health Organization, Geneva, v. 83, n. 6, p. 418-426, 2005.

BARBOSA, A. P.; CUNHA, A. J. L. A. Neonatal and pediatric intensive care in Rio de Janeiro State, Brazil: an analysis of bed distribution, 1997 and 2007. Cadernos de Saúde Pública, Rio de Janeiro, v. 27, p. s263-s271, 2011. Suplemento 2.

BARDIN, L. Análise de conteúdo. 3. ed. Lisboa: Edições 70, 2004.

BARROS, F. C. et al. Global report on preterm birth and stillbirth ( 3 of 7 ): evidence for effectiveness of interventions. BMC Pregnancy and Childbirth, London, v. 10, p. S3, 2010a. Supplement.

BARROS, F. C. et al. Recent trends in maternal, newborn, and child health in Brazil: progress toward Millennium Development Goals 4 and 5 . American Journal of Public Health, Washington, DC, v. 100, n. 10, p. 1877-1889, 2010 .

BETTIOL, H. A demanda por um novo pediatra. Ciência \& Saúde Coletiva, Rio de Janeiro, v. 15, n. 2, p. 330-332, 2010.

CALDEIRA, A. P.; OLIVEIRA, R. M. D.; RODRIGUES, O. A. Qualidade da assistência materno-infantil em diferentes modelos de atenção primária. Ciência \& Saúde Coletiva, Rio de Janeiro, v. 15, p. 3139-3147, 2010. Suplemento 2.

CFM - CONSELHO FEDERAL DE MEDICINA. Resolução CFM n ${ }^{0}$ 1.957, de 15 de dezembro de 2010. Diário Oficial da União, Brasília, DF, 6 jan. 2011. Seção I, p. 79. Disponível em: <http:// www.portalmedico.org.br/resolucoes/CFM/2010/ 1957_2010.htm >. Acesso em: 20 maio 2012.

CFM - CONSELHO FEDERAL DE MEDICINA. Resolução CFM nº 2.013, de 16 de abril de 2013. Adota as normas éticas para a utilização das técnicas de reprodução assistida, anexas à presente resolução, como dispositivo deontológico a ser seguido pelos médicos e revoga a Resolução CFM n ${ }^{0}$ 1.957/10. Diário Oficial da União, Brasília, DF, 9 maio 2013. Seção I, p. 119. Disponível em: <http://www.portalmedico.org.br/resolucoes/ CFM/2013/2013_2013.pdf >. Acesso em: 3 ago. 2013. 
COSTA, M. C. N. et al. Mortalidade infantil no Brasil em períodos recentes de crise econômica. Revista de Saúde Pública, São Paulo, v. 37, n. 6, p. 699-706, 2003.

CRUZ, S. A. et al. Evolution of inequalities in mortality in Salvador, Bahia State, Brazil, 1991/2006. Cadernos de Saúde Pública, Rio de Janeiro, v. 27, p. S176-S184, 2011. Suplemento 2.

DATASUS. População residente: Paraná. Brasília, DF, 2011. Disponível em: <http://tabnet.datasus. gov.br/cgi/tabcgi.exe?ibge/cnv/poppr.def >. Acesso em: 8 mar. 2011.

DOMINGUES, R. M. S. M. et al. Avaliação da adequação da assistência pré-natal na rede SUS do Município do Rio de Janeiro, Brasil. Cadernos de Saúde Pública, Rio de Janeiro, v. 28, n. 3, p. 425437, 2012.

DUARTE, C. M. Reflexos das políticas de saúde sobre as tendências da mortalidade infantil no Brasil: revisão da literatura sobre a última década. Cadernos de Saúde Pública, Rio de Janeiro, v. 23, n. 7, p. 1511-1528, 2007.

DUNN, J.; FERRI, C. P. Epidemiological methods for research with drug misusers: review of methods for studying prevalence and morbidity. Revista de Saúde Pública, São Paulo, v. 33, n. 2, p. 206-215, 1999.

FERREIRA, R. A. et al. Análise espacial da vulnerabilidade social da gravidez na adolescência. Cadernos de Saúde Pública, Rio de Janeiro, v. 28, n. 2, p. 313-323, 2012.

FONTANELLA, B. J.; RICAS, J.; TURATO, E. R. Amostragem por saturação em pesquisas qualitativas em saúde: contribuições teóricas. Cadernos de Saúde Pública, Rio de Janeiro, v. 24, n. 1, p. 17-27, 2008.

GABANI, F. L.; SANT'ANNA, F. H. M.; ANDRADE, S. M. Caracterização dos nascimentos vivos no município de Londrina (PR) a partir dos dados do SINASC. Ciência, Cuidado e Saúde, Maringá, v. 9, n. 2, p. 205-213, 2010.
GIGLIO, M. R. P.; FRANÇA, E.; LAMOUNIER, J. A. Avaliação da qualidade da assistência ao parto normal. Revista Brasileira de Ginecologia e Obstetrícia, Rio de Janeiro, v. 33, n. 10, p. 297-304, 2011.

GIL, C. R. R.; MARTIN, G. B.; GUTIERREZ, P. R. A organização dos serviços de saúde de Londrina e região: uma experiência concreta do processo de municipalização. In: ANDRADE, S. M.; SOARES, D. A.; CORDONI JR, L. (Org.). Bases da saúde coletiva. Londrina: EDUEL, 2001. p. 61-91.

GOMES, M. A. et al. Assistência e mortalidade neonatal no setor público do Município do Rio de Janeiro, Brasil: uma análise do período 1994/200o. Cadernos de Saúde Pública, Rio de Janeiro, v. 21, n. 4, p. 1269-1277, 2005.

GOULART, L. M. H. D. F.; SOMARRIBA, M. G.; XAVIER, C. C. A perspectiva das mães sobre o óbito infantil: uma investigação além dos números. Cadernos de Saúde Pública, Rio de Janeiro, v. 21, n. 3, p. 715-723, 2005.

GRANER, V. R.; BARROS, S. M. Complicações maternas e ocorrências neonatais associadas às gestações múltiplas resultantes de técnicas de reprodução assistida. Revista da Escola de Enfermagem da USP, São Paulo, v. 43, n. 1, p. 103109, 2009.

HERNANDEZ, A. R. et al. Análise de tendências das taxas de mortalidade infantil e de seus fatores de risco na cidade de Porto Alegre, Rio Grande do Sul, Brasil, no período de 1996 a 2008. Cadernos de Saúde Pública, Rio de Janeiro, v. 27, n. 11, p. 2188-2196, 2011.

HOLCMAN, M. M.; LATORRE, M. R. D. O.; SANTOS, J. L. F. Evolução da mortalidade infantil na região metropolitana de São Paulo, 1980-200o. Revista de Saúde Pública, São Paulo, v. 38, n. 2, p.18o-186, 2004 .

JUNQUEIRA, T. S. et al. As relações laborais no âmbito da municipalização da gestão em saúde e os dilemas da relação expansão/precarização do trabalho no contexto do SUS. Cadernos de Saúde Pública, Rio de Janeiro, v. 26, n. 5, p. 918-928, 2010. 
LIMA, L. D.; ANDRADE, C. L. T. Condições de financiamento em saúde nos grandes municípios do Brasil. Cadernos de Saúde Pública, Rio de Janeiro, v. 25, n. 10, p. 2237-2248, 2009.

LONDRINA. Secretaria Municipal de Saúde. Conselho Municipal de Saúde. Relatório anual de gestão da saúde 2011. Londrina, 2011. Disponível em: <http://www.londrina.pr.gov.br/dados/images/ stories/Storage/sec_saude/relatorios_gestao/ relatorio_gestao_2011.pdf >. Acesso em: 2 ago. 2013.

MACHADO, M. M. et al. A community perspective on changes in health related to diarrhea in northeastern Brazil. Food and Nutrition Bulletin, Boston, v. 32, n. 2, p. 103-111, 2011.

MARTIN, J. A. Preterm births: United States, 2007. Morbidity and Mortality Weekly Report: surveillance summaries, Atlanta, v. 6o, n. 1, p. 78 79, 2011. Supplement.

MATHIAS, T. A. F.; ASSUNÇÃO, A. N.; SILVA, G. F. Óbitos infantis investigados pelo Comitê de Prevenção da Mortalidade Infantil em região do Estado do Paraná. Revista da Escola de Enfermagem da USP, São Paulo, v. 42, n. 3, p. 445453, 2008.

MENDOZA-SASSI, R. A. et al. Diferenças no processo de atenção ao pré-natal entre unidades da Estratégia Saúde da Família e unidades tradicionais em um município da Região Sul do Brasil. Cadernos de Saúde Pública, Rio de Janeiro, v. 27, n. 4, p. 787-796, 2011.

NATIONS, M. K. Infant death and interpretive violence in Northeast Brazil: taking bereaved Cearense mothers' narratives to heart. Cadernos de Saúde Pública, Rio de Janeiro, v. 24, n. 10, p. 2239-2248, 2008.

NORONHA, R. P. O desenvolvimento federativo do SUS e as novas modalidades institucionais de gerência das unidades assistenciais. In: SANTOS, N. R.; AMARANTE, P. D. C. (Org.). Gestão pública e relação público privado na saúde. Rio de Janeiro: Cebes, 2011. p. 24-47.
OLIVEIRA, E. F. V. D.; GAMA, S. G. N. D.; SILVA, C. M. F. P. D. Gravidez na adolescência e outros fatores de risco para mortalidade fetal e infantil no Município do Rio de Janeiro, Brasil. Cadernos de Saúde Pública, Rio de Janeiro, v. 26, n. 3, p. 567578, 2010.

OLIVEIRA, M. I. C. et al. Qualidade da assistência ao trabalho de parto pelo Sistema Único de Saúde, Rio de Janeiro (RJ), 1999-2001. Revista de Saúde Pública, São Paulo, v. 42, n. 5, p. 895-902, 2008.

SANTOS, A. L. D.; ROSENBURG, C. P.; BURALLI, K. O. Histórias de perdas fetais contadas por mulheres: estudo de análise qualitativa. Revista de Saúde Pública, São Paulo, v. 38, n. 2, p. 268-276, 2004 .

SANTOS, H. G. et al. Risk factors for infant mortality in a municipality in southern Brazil: a comparison of two cohorts using hierarchical analysis. Cadernos de Saúde Pública, Rio de Janeiro, v. 28, n. 1o, p. 1915-1926, 2012.

SILVA, A. C. M. A. et al. Perspectivas de médicos do Programa Saúde da Família acerca das linhas de cuidado propostas pela Agenda de Compromissos para a Saúde Integral da Criança e Redução da Mortalidade Infantil. Cadernos de Saúde Pública, Rio de Janeiro, v. 25, n. 2, p. 349-358, 2009a.

SILVA, A. M. R. et al. Fatores de risco para nascimentos pré-termo em Londrina, Paraná, Brasil. Cadernos de Saúde Pública, Rio de Janeiro, v. 25, n. 10, p. 2125-2138, 2009b.

SILVEIRA, M. F. et al. Increase in preterm births in Brazil: review of population-based studies. Revista de Saúde Pública, São Paulo, v. 42, n. 5, p. 957-964, 2008.

SOUSA, J. R. P. D.; NATIONS, M. Múltiplos olhares sobre a mortalidade infantil no Ceará, Brasil. Cadernos de Saúde Pública, Rio de Janeiro, v. 27, n. 2, p. 26o-268, 2011.

SOUZA, S. N. D. H. $O$ aleitamento materno na perspectiva da vulnerabilidade programática. 2010. Tese (Doutorado em Enfermagem) - Escola de Enfermagem de Ribeirão Preto da Universidade de São Paulo, Ribeirão Preto, 2010. 
TAKEMURA, N. S.; ANDRADE, S. M. Meningite por haemophilus influenzae tipo b em cidades do Estado do Paraná, Brasil. Jornal de Pediatria, Rio de Janeiro, v. 77, n. 5, p. 387-392, 2001.

TOMÉ, E. A.; LATORRE, M. R. D. O. Tendências da mortalidade infantil no Município de Guarulhos: análise do período de 1971 a 1998. Revista

Brasileira de Epidemiologia, São Paulo, v. 4, n. 3, p. 153-167, 2001.

TURATO, E. R. Métodos qualitativos e quantitativos na área da saúde: definições, diferenças e seus objetos de pesquisa. Revista de Saúde Pública, São Paulo, v. 39, n. 3, p. 507-514, 2005.

VENÂNCIO, S. I.; PAIVA, R. D. O processo de implantação dos Comitês de Investigação do óbito Infantil no Estado de São Paulo. Revista Brasileira de Saúde Materno Infantil, Recife, v. 10, n. 3, p. 369-375, 2010.

VERANI, J. R. et al. Prevention of perinatal group B streptococcal disease: revised guidelines from CDC, 2010. MMWR, Recommendations and reports: Morbidity and mortality weekly report, Atlanta, v. 59, p. 1-36, Nov. 2010.
VERMELHO, L. L.; COSTA, A. J. L.; KALE, P. L. Indicadores de saúde. In: MEDRONHO, R. A. et al. (Org.). Epidemiologia. 2. ed. São Paulo: Atheneu, 2009. p. 31-82.

VICTORA, C. G. et al. Evidence for protection by breast-feeding against infant deaths from infectious diseases in Brazil. The Lancet, London, v. 2, n. 8554, p. 319-322, 1987.

VICTORA, C. G. et al. Socio-economic and ethnic group inequities in antenatal care quality in the public and private sector in Brazil. Health Policy and Planning, London, v. 25, n. 4, p. 253-261, 2010. VICTORA, C. G. et al. Maternal and child health in Brazil: progress and challenges. The Lancet, London, v. 377, n. 978o, p. 1863-1876, 2011.

ZANINI, R. R. et al. Infant mortality trends in the State of Rio Grande do Sul, Brazil, 1994-2004: a multilevel analysis of individual and community risk factors. Cadernos de Saúde Pública, Rio de Janeiro, v. 25, n. 5, p. 1035-1045, 2009. 